\title{
¿Qué repercusión a nivel fisiológico puede tener realizar una carrera de ultratrail? Análisis de la alteración de biomarcadores de daño cardíaco y muscular en corredores amateurs de ultratrail
}

\author{
Claudia Collado-Andrés \\ al290690@uji.es \\ Bárbara Hernando \\ hernandb@uji.es \\ Carlos Hernando \\ hernando@uji.es \\ Conrado Martínez-Cadenas \\ ccadenas@med.uji.es
}

\section{Resumen}

La participación en ultratrails ha incrementado significativamente su popularidad en los últimos años. Debido a las características extremas de estas pruebas y a las consecuencias que pueden tener sobre la salud de los participantes, es necesario investigar determinados parámetros para conocer más a fondo su repercusión fisiológica. El objetivo principal de este trabajo es ver la evolución de los parámetros bioquímicos y el tiempo que tardan en normalizarse, teniendo en cuenta el rendimiento deportivo de los corredores. Para ello, de un total de 33 corredores ( 28 hombres y 5 mujeres) que finalizaron la Penyagolosa Trails CSP115, se recogieron muestras de sangre en la salida, en la meta, así como a las $72 \mathrm{~h}$ y $168 \mathrm{~h}$ tras finalizar la carrera. Los corredores fueron clasificados como rápidos (tiempo final $\leq$ media) o lentos (tiempo final > media). Se realizó un análisis de la evolución de diferentes biomarcadores relacionados con el daño muscular y cardíaco, teniendo en cuenta el rendimiento deportivo de los corredores. Los resultados muestran que existe una alteración fisiológica aguda tras la realización de una ultratrail, aunque los valores de todos los parámetros fisiológicos analizados se normalizan en la fase de recuperación (hasta $168 \mathrm{~h}$ poscarrera). Así pues, realizar una fase de recuperación larga tras una ultratrail es recomendable para la normalización de los valores antes de volver a la rutina de entrenamientos. Además, los corredores con un mejor rendimiento deportivo 
presentan una mayor alteración fisiológica, seguramente debido al mayor esfuerzo físico al que someten su cuerpo.

Palabras clave: ultratrail, salud, rabdomiolisis, daño cardíaco, recuperación.

\section{Abstract}

The participation in ultratrails has significantly increased its popularity in recent years. Due to the extreme characteristics of ultratrail races and to the consequences in runners' health, it is necessary to analyze certain healthrelated parameters in order to know the effect of running an ultratrail on the human body. The main objective of this work is to see the evolution of the biochemical parameters and the time it takes to normalize taking into account the athletic performance of the runners. To do this, blood samples were collected from 33 runners (28 men and 5 women) who finished the Penyagolosa Trails CSP115, at four different moments: on the starting line, after crossing the finish line, 72 hours after finishing the race and 168 hours post-race. Runners were classified according to their performance as fast (final time < average) and slow runners (final time > average). The analyses were focused on analyzing the evolution of different biomarkers related to muscle and cardiac damage, taking into account the athletic performance of runners. Our results show that there are acute physiological changes after running an ultratrail race, although all parameters analyzed are completely normalized during the recovery period (until 168 hours post-race). Therefore, performing a complete recovery period after running an ultratrail prior to start training sessions again is highly recommended. In addition, a higher physiological alteration has been observed in runners who present a better performance (faster runners), probably because of their bodies has been subjected to a higher physical effort.

Keywords: Ultratrail, health, rhabdomyolysis, heart damage, recovery.

\section{Introducción}

El término ultratrail se utiliza para denominar carreras de larga distancia englobadas dentro de la modalidad de trail running, la cual abarca toda carrera natural (carreras a pie que discurran por terreno natural sin más de un $30 \%$ de asfalto como referencia máxima). Así pues, una ultratrail se define como una carrera natural con una distancia superior que la de un maratón estándar (42,195 km) (Hoffman 2016). Típicamente, las carreras de ultratrail suelen realizarse por montaña $\mathrm{y}$, por lo tanto, deben tener desniveles, tanto positivos como negativos, que incrementan sustancialmente la exigencia de la carrera. Estas carreras de ultratrail pueden estar organizadas en etapas o de manera continua, con un límite máximo de tiempo para realizarlas. Otra de las características de estas carreras es que son de carácter individual en semiautosuficiencia. Esto implica que cada corredor tiene que tener la capacidad de organizar su propia supervivencia entre avituallamientos, tanto desde el punto de vista alimentario como de seguridad o de vestuario. Por lo tanto, salvo en caso de accidente - emergencia, la asistencia personal está autorizada solo en los puntos de avituallamiento. Por este motivo, la organización suele obligar a los participantes a llevar un mínimo de material por la seguridad de los corredores, que puede ser 
revisado en los controles y que implica la descalificación si no se cumplen los requisitos (Knechtle y Nikolaidis 2018).

Con el objetivo de investigar acerca del impacto que tiene realizar una carrera de ultratrail en el organismo, recientemente se han llevado a cabo estudios en el ámbito de la medicina deportiva centrados en analizar los cambios que se producen en los valores de diferentes biomarcadores tras haber realizado una ultratrail, respecto a los valores basales del corredor. La mayoría de los estudios publicados hasta el momento coinciden en que realizar una carrera de ultratrail provoca alteraciones funcionales importantes. Las partes del cuerpo del corredor que sufren especialmente tras un ultratrail son los músculos de las piernas y el corazón (Khodaee, Spittler, VanBaak, Changstrom y Hill 2015; Kim, Lee y Kim 2007). De hecho, diversos estudios muestran un incremento significativo de marcadores hematológicos relacionados con el daño muscular y cardíaco como, por ejemplo, la enzima creatina-quinasa (CK), la enzima lactato deshidrogenasa (LDH) o la troponina.

Debido a las condiciones de la carrera, como son correr de noche por senderos montañosos no señalizados a temperaturas extremas y en régimen individual de semiautosuficiencia, son consideradas pruebas de alto riesgo. Los corredores deben enfrentarse a duras subidas, a bajadas muy técnicas y frecuentemente a una o incluso dos noches de carrera sin dormir. Realizar estas carreras supone un desgaste muscular importante y, por lo tanto, pueden aparecer calambres y dolor muscular, entre otras complicaciones, que se concentran especialmente en las piernas. Además, realizar esfuerzos físicos de esta magnitud puede provocar alteraciones fisiológicas más severas que pueden afectar a la actividad del corazón del corredor de ultratrail, pudiendo causar la muerte súbita. La alteración del ritmo cardíaco unido a la fatiga muscular y la deshidratación pueden llegar a causar mareos e incluso pérdidas de concien cia derivadas del síndrome del colapso (Holtzhausen et al. 1994)

Sin embargo, los estudios centrados en analizar el daño cardíaco señalan que las alteraciones observadas no suelen inducir un fallo del miocárdico (Khodaee, Spittler, VanBaak, Changstrom y Hill 2015), sino que realizar un ejercicio aeróbico prolongado en el medio rural de forma moderada parece aumentar la salud cardíaca (Khodaee, Spittler, VanBaak, Changstrom y Hill 2015; Kim et al. 2012; Son, Lee, Chae y Kim 2015). Además, los valores anormales de los biomarcadores cardíacos parecen normalizarse a partir de las 24 horas de descanso (Christensen et al. 2017). Así pues, seguramente, este daño cardíaco es transitorio y no estaría asociado normalmente a una alteración cardiaca relevante para el corredor. Estos estudios también apuntan a que el daño en los miocitos es significativamente mayor cuanto menos preparados están los corredores (Khodaee, Spittler, VanBaak, Changstrom y Hill 2015). Con estos resultados se pone de manifiesto la importancia que supone para el organismo realizar este tipo de carreras y se enfatiza la necesidad de una adecuada preparación previa.

Esta investigación se enmarca dentro del proyecto de investigación Penyagolosa Trails Saludable (PTS), organizado por el Servicio de Deportes de la Universitat Jaume I de Castellón, el club de montaña Desert Amunt y Penyagolosa Trails y financiado por la Univesitat Jaume I, la Fundación de Hospitales Vithas-Nisa y el Villarreal CF. Dicho proyecto surge en el 2014 con el objetivo de poner en marcha una investigación pionera en España, con la que obtener datos relacionados con la salud de los corredores por medio de la medición de factores psicológicos, de hábitos de entrenamiento y de parámetros físicos y fisiológicos, como la evolución del peso, la fuerza muscular, la tensión arterial y las analíticas sanguíneas y de orina. Para ello, en este proyecto se monitorizaron corredores de ultratrail participantes en la edición del 2015 de la Castelló-Penyagolosa Trail (CSP-115). 
La CSP-115 consta de $119 \mathrm{~km}$, con un desnivel acumulado de subida de 5600 metros y de bajada de 4400 metros, que deben recorrerse en régimen de semiautosuficiencia en un tiempo total máximo de 30 horas. La carrera se inicia en Castellón de la Plana y finaliza en el santuario de Sant Joan de Penyagolosa. Durante el recorrido, existen diferentes avituallamientos donde los corredores pueden reponer fuerzas y recibir apoyo externo.

La hipótesis inicial que nos planteamos al llevar a cabo esta investigación era que los corredores que finalizasen la CSP-115 iban a sufrir alteraciones fisiológicas importantes, pero que estas iban a ser transitorias, ya que después de un periodo de recuperación alcanzarían valores de normalidad. Además, pensábamos que, debido al nivel de entrenamiento y al menor tiempo realizando la carrera, los corredores clasificados como rápidos iban a sufrir menores daños tanto musculares como cardíacos. Así pues, los corredores lentos tendrían, para todos los biomarcadores analizados, una mayor diferencia entre el valor de referencia basal y el valor obtenido en las muestras recogidas en meta.

Por lo tanto, el objetivo principal de este trabajo es determinar si existe una alteración fisiológica aguda tras realizar una ultratrail y si esta es transitoria de forma que los corredores vuelven a presentar valores similares a los basales tras una semana de recuperación. Además, se evaluará si las alteraciones fisiológicas sufridas y la capacidad de recuperación de los corredores depende de su rendimiento deportivo, de forma que se comparará cómo varían los parámetros durante el transcurso de la carrera y el periodo de recuperación entre corredores rápidos y lentos.

\section{Métodos}

Para realizar la captación de voluntarios participantes en el estudio, inicialmente se envió un correo informativo a todos los inscritos a la carrera (600 corredores). De los que contestaron al correo, se descartaron aquellos que no cumplieran los criterios de inclusión establecidos. Los criterios de inclusión fueron: ser mayor de edad, no padecer ninguna enfermedad cardíaca y haber realizado anteriormente una ultramaratón.

El objetivo era tener una población de 50 corredores con un número equiparable entre hombres y mujeres. Por eso, se incluyeron en el estudio todas las mujeres que contestaron afirmativamente $(\mathrm{N}=6)$, mientras que la selección de los hombres se realizó de forma aleatoria $(\mathrm{N}=44)$. De los 50 corredores que tomaron la salida de la carrera, llegaron a meta 33 (34\% de abandono). Así pues, la población de estudio en este trabajo de investigación fue de 33 corredores finishers (28 hombres y 5 mujeres) de la Penyagolosa Trails CSP 2015, con un rango de edad de entre 22 y 55 (media $39,30 \pm 7,56$ años).

Se utilizó una encuesta en línea para recoger datos sobre sus hábitos de entrenamiento, medicación habitual y antecedentes médicos. Previamente a realizar el trabajo de campo, se realizó una reunión informativa a las 50 personas seleccionadas como participantes del estudio con el objetivo de presentar la finalidad del proyecto y explicar las diferentes pruebas y datos que iban a ser recogidos. Para esta investigación, todos los participantes fueron sometidos a los mismos exámenes bajo las mismas condiciones.

Se recogieron un total de 4 muestras de sangre de todos los participantes del estudio. Los momentos de extracción de muestras de sangre fueron: en la feria del corredor realizada el día previo a la salida (FERIA), tras finalizar la carrera (META), $72 \mathrm{~h}$ tras 
haber finalizado la carrera y $168 \mathrm{~h}$ poscarrera. Las muestras de sangre se tomaron mediante venopunción de venas antecubitales utilizando tubos BD Vacutainer PST II. Las muestras fueron trasportadas a $4{ }^{\circ} \mathrm{C}$ hasta el laboratorio del Hospital Vithas Nisa Jaime I de Castellón. Todos los resultados bioquímicos sanguíneos poscarrera fueron ajustados por medio del índice de Kargotich (Kargotich et al. 1997) y teniendo en cuenta las modificaciones del volumen plasmático debidas a la deshidratación, de acuerdo con el método de Dill y Costill (1974). De las muestras extraídas de sangre se realizaron análisis de marcadores bioquímicos de daño muscular y cardíaco [CK, LDH, la troponina y la proteína c-reactiva (PCR)].

Los análisis estadísticos se han realizado utilizando el programa estadístico SPSS, versión 23. Inicialmente, se comprobó la distribución normal de las variables de estudio mediante el test de normalidad Kolgomorov-Smirnoff. Para comparar los valores de una variable cuantitativa entre determinaciones sucesivas dentro del mismo grupo se empleó el test $t$ de Student para muestras pareadas. Para comparar los valores de una variable cuantitativa entre dos grupos de muestras en un momento concreto de recogida de datos se empleó el test ANOVA de un factor. Para considerar un resultado como estadísticamente significativo, el p-valor bilateral obtenido de los análisis estadísticos debía ser menor de 0,05.

\section{Resultados}

Este trabajo de investigación se centra en analizar la evolución de ciertos marcadores relacionados con diferentes alteraciones fisiológicas en corredores de ultratrail, teniendo en cuenta el rendimiento del corredor. De ahí que los corredores que finalizaron la carrera (finishers) fueran separados en dos grupos según su tiempo de carrera: rápidos (corredores con un tiempo final inferior a la media de todos los corredores del estudio) y lentos (corredores con un tiempo final superior a la media).

Los marcadores analizados para determinar la existencia de daño muscular y cardíaco después de realizar la CSP-115 son los niveles de LDH, CK, PCR y troponina en sangre. Los resultados muestran que no existen diferencias significativas en los niveles de LDH en sangre en ninguno de los momentos de toma de muestra entre corredores rápidos y lentos. Sin embargo, sí que se observa un incremento significativo de los niveles de LDH en las muestras recogidas tras cruzar la línea de meta respecto a los niveles basales, independientemente del rendimiento del corredor ( $p$-value $<0,001$ en ambos grupos de corredores). Se observa que la normalización de los valores es mucho más rápida en corredores lentos que en rápidos (figura 1). 


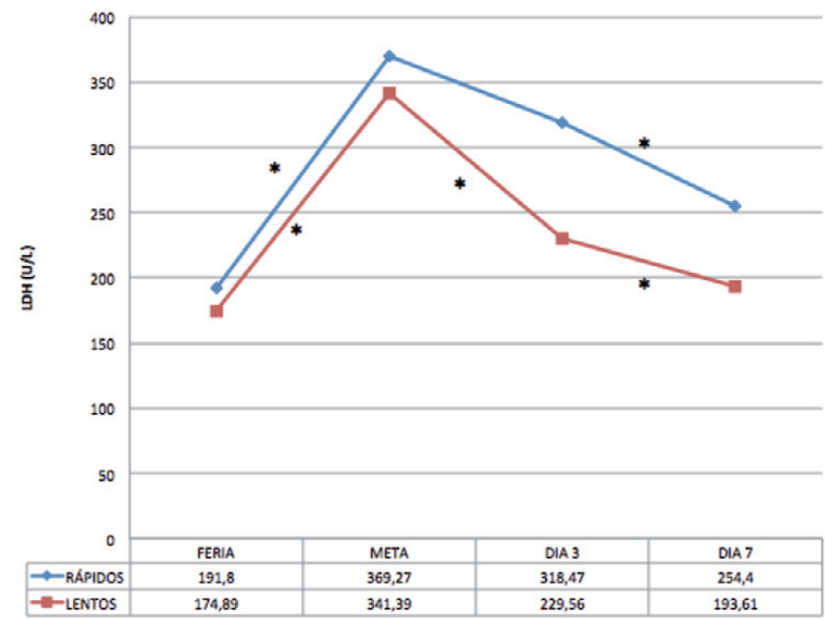

Figura 1. Representación gráfica de la evolución de los niveles de LDH en sangre. El asterisco $\left({ }^{*}\right)$ indica que existen diferencias significativas entre dos momentos de recogida de muestras dentro del grupo. El nivel de significación en todas las comparaciones realizadas es inferior a 0,01.

Otro marcador relacionado con el daño muscular es el nivel de CK en sangre. Tanto los corredores lentos como los rápidos parecen sufrir una destrucción muscular tras realizar la CSP-115, ya que se incrementan los niveles de CK en meta respecto a los valores basales ( $p$-value $<0,001$ tanto en corredores rápidos como en lentos). Sin embargo, es muy llamativa la diferencia de concentración sanguínea de CK entre corredores rápidos y lentos en meta. Posiblemente, a mayor intensidad del esfuerzo, el daño muscular incrementa y, por ello, los corredores con mayor rendimiento tienen hasta 7 veces más incrementado este parámetro respecto al valor basal. Aunque es cierto que esta alteración también parece ser transitoria, ya que al séptimo día se normalizan los niveles de $\mathrm{CK}$ en sangre tanto en corredores rápidos como en lentos (figura 2).

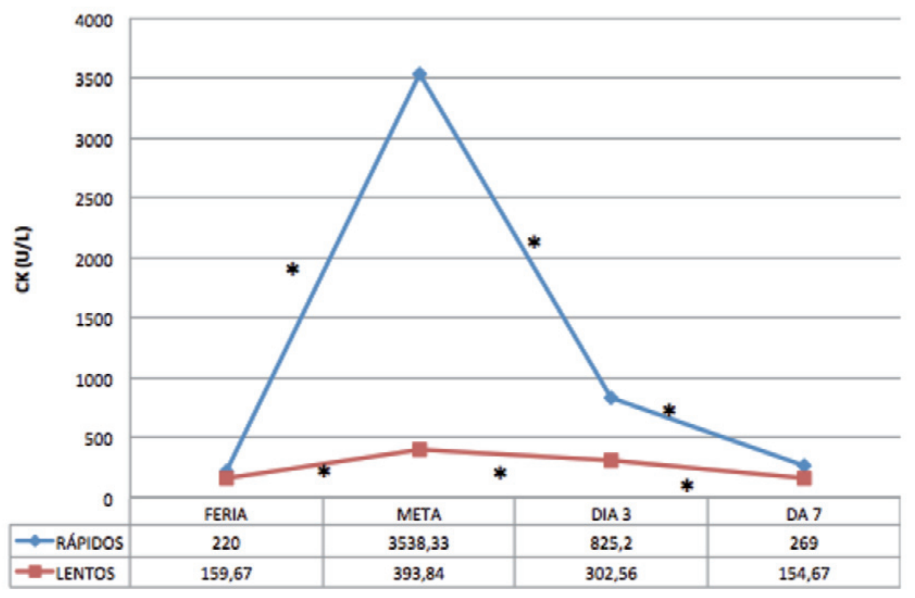

Figura 2. Representación gráfica de la evolución de los niveles de CK en sangre. El asterisco $\left({ }^{*}\right)$ indica que existen diferencias significativas entre dos momentos de recogida de muestras dentro del grupo. El nivel de significación en todas las comparaciones realizadas es inferior a 0,01. 
Asimismo, los resultados muestran que existen diferencias significativas en los niveles de troponina en sangre en meta entre los dos grupos de corredores ( $p$-value $=0,027$ ). También existen diferencias significativas al comparar los valores de este marcador dentro del mismo grupo entre los distintos puntos de toma de muestras (feria vs. meta: $p$-value $=0,002$ en corredores rápidos y $p$-value $=0,001$ en lentos; meta vs. día 3 poscarrera: $p$-value $=0,001$ en corredores rápidos, y $p$-value $=0,001$ en lentos). Los niveles de troponina son mucho más elevados en meta debido al estrés al que el corazón se ha visto sometido, pero estos valores se normalizan al tercer día de recuperación. Sin embargo, tal y como se muestra en la figura 3 , los niveles de troponina en sangre vuelven a incrementarse al séptimo día de recuperación respecto a los niveles basales. Esto puede ser debido a que los corredores no han cumplido una semana de reposo completo y han retomado el entrenamiento.

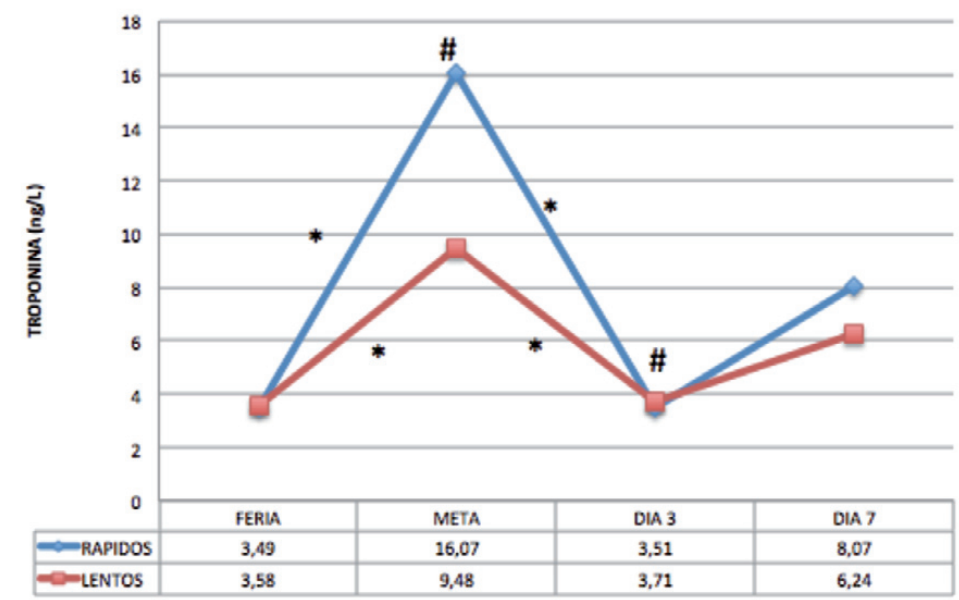

Figura 3. Representación gráfica de la evolución de los niveles de troponina en sangre.

El asterisco $\left({ }^{*}\right)$ indica que existen diferencias significativas entre dos momentos de recogida de muestras dentro del grupo. La almohadilla (\#) indica que existen diferencias significativas entre rápidos y lentos en ese punto.

Por último, se analizaron los cambios en los niveles de PCR en sangre. Los análisis estadísticos muestran que hay diferencias estadísticamente significativas al comparar los grupos antes de iniciar la carrera $(p$-value $=0,031)$. Los corredores lentos incrementan en mayor medida los niveles de PCR tras realizar una ultratrail, aunque se observa que el séptimo día los valores han vuelto a la normalidad. Los corredores rápidos, sin embargo, tienen un menor incremento del nivel de PCR en sangre tras realizar la CSP-115 respecto al basal, aunque parece que los valores tardan más en normalizarse (figura 4). Para entender la evolución de los niveles de PCR en sangre primero se debe puntualizar que este es un marcador cuyos valores son más lentos en ascender a nivel analítico, en comparación con los otros biomarcadores de daño cardíaco y muscular analizados. Esto explicaría por qué el simple hecho de llevar más tiempo realizando la carrera implica que los valores de PCR en sangre tras cruzar la meta de la CSP-115 sean más altos en corredores lentos que en rápidos. 


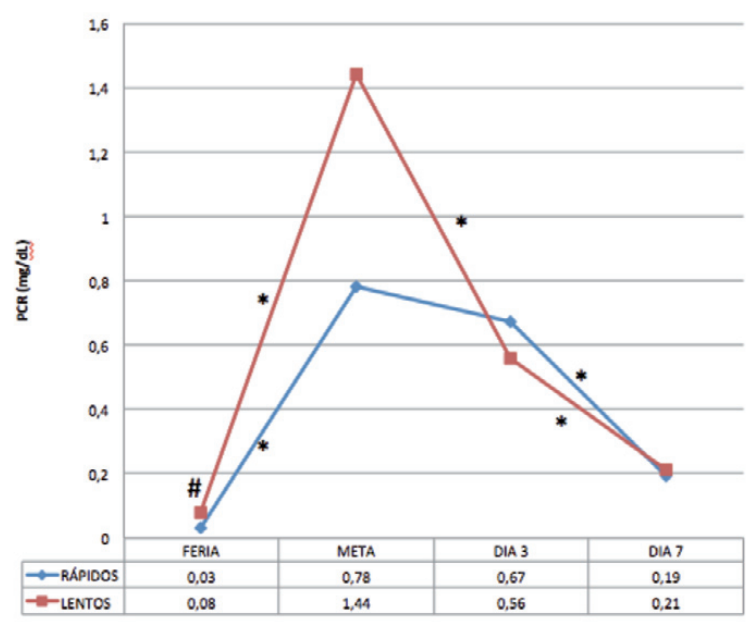

Figura 4. Representación gráfica de la evolución de los niveles de PCR en sangre. El asterisco $\left({ }^{*}\right)$ indica que existen diferencias significativas entre dos momentos de recogida de muestras dentro del grupo. La almohadilla (\#) indica que existen diferencias significativas entre rápidos y lentos en ese punto.

\section{Discusión, conclusiones e implicaciones prácticas}

Este estudio de investigación se centra en analizar el impacto fisiológico que tiene realizar una ultratrail. Para ello, se recogen datos de diferentes parámetros fisiológicos en 33 corredores que finalizaron la CSP-115, una carrera de ultratrail con un recorrido de $118 \mathrm{~km}$. Con el objetivo de analizar la evolución de los diferentes parámetros incluidos en el trabajo, tanto durante la carrera como en el proceso de recuperación de la misma, la toma de muestras se realiza antes de la carrera (feria) y tras cruzar la meta (meta), así como a las $72 \mathrm{~h}$ y 164 h tras haber finalizado la carrera.

Debido a que la preparación previa y el rendimiento deportivo parecen influir en las alteraciones fisiológicas que sufre el corredor (Khodaee, Spittler, VanBaak, Changstrom y Hill 2015), el total de la población se dividió en dos grupos de estudio. Los corredores clasificados como rápidos $(\mathrm{N}=15)$ eran aquellos que finalizaban la prueba con un tiempo de carrera inferior a la media de todos los corredores y los corredores clasificados como lentos $(\mathrm{N}=18)$ eran aquellos con un tiempo de finalización de carrera superior a la media.

Existen estudios previos que han analizado marcadores relacionados con el daño muscular y cardíaco para ver las alteraciones que sufre el organismo de un corredor al realizar una carrera de ultratrail (Baird, Graham, Baker y Bickerstaff 2012; Khodaee, Spittler, VanBaak, Changstrom y Hill 2015; Kim et al. 2012; Son, Lee, Chae y Kim 2015). Todos estos estudios coinciden en indicar que existe un aumento exponencial de los niveles de troponina, CK, LDH y PCR en sangre después de haber realizado una carrera de estas características. Nuestros resultados, además de confirmar estas alteraciones previamente descritas, señalan que los valores de estos marcadores fisiológicos se normalizan a partir del tercer día de recuperación tras realizar la carrera.

Como se puede observar, son muchos los estudios previos que han analizado con detenimiento las alteraciones de biomarcadores fisiológicos relacionados con el daño muscular y cardíaco sufridas por corredores de ultratrail (Baird, Graham, Baker y Bickerstaff 2012; Joslin et al. 2014; Knechtle y Nikolaidis 2018). Sin embargo, este 
estudio es pionero a nivel nacional ya que, además de analizar los cambios fisiológicos que se producen en el corredor durante la carrera, también se centra en investigar la evolución de los niveles de ciertos biomarcadores fisiológicos durante la semana de recuperación de la carrera con el objetivo de conocer si estas alteraciones son transitorias y el tiempo que necesita el organismo del corredor para recuperarse.

Este trabajo de investigación no está exento de limitaciones. Una de las mayores limitaciones del proyecto es el tamaño de nuestra población, el cual, además, se reduce debido a que no todos los corredores finalizan la carrera CSP-115 (aspecto con el que ya se contaba al diseñar el trabajo debido a las características de la carrera). Nuestro objetivo principal era estudiar la evolución de ciertos parámetros fisiológicos tras realizar la carrera de ultratrail y en el proceso de recuperación. De ahí que el presupuesto asociado al proyecto se destinara a ampliar el número de muestras recogidas por voluntario y no en ampliar el número de voluntarios en el estudio. Por otro lado, debido a que únicamente se incluyen 5 mujeres en el proyecto, no se han podido realizar comparaciones en la evolución de los parámetros fisiológicos y antropométricos entre hombres y mujeres. De ahí que se haya puesto en marcha una segunda fase de este proyecto de investigación, Proyecto Penyagolosa Trails Saludable Women, centrado en evaluar las diferencias existentes en la repercusión de un trabajo de larga duración entre los hombres y las mujeres.

Así pues, los resultados obtenidos en este proyecto de investigación corroboran la hipótesis inicialmente planteada, ya que se ha determinado que existe una alteración fisiológica aguda tras realizar la CSP-115 y que los valores vuelven a la normalidad tras una semana de recuperación. Sin embargo, aunque inicialmente pensábamos que los corredores rápidos iban a sufrir un menor daño muscular y cardíaco debido a que están más preparados para realizar carreras de estas características, los resultados muestran que son los corredores rápidos los que tienen más elevados los niveles de biomarcadores asociados con destrucción muscular y daño cardíaco. Seguramente, debido a que están más preparados físicamente, son capaces de llevar a su cuerpo a rendir hasta límites más extremos.

Desde un punto de vista práctico, el proyecto Penyagolosa Trail Saludable intenta aportar información valiosa relacionada con la dosificación del esfuerzo en carreras de estas características tanto a los corredores como a los entrenadores. Por una parte, las conclusiones extraídas de este trabajo pueden aplicarse a la necesidad de crear protocolos de mantenimiento de la hidratación con el objetivo de evitar un mayor daño fisiológico, así como entrenamientos centrados en disminuir los daños asociados a realizar este tipo de carreras de elevada exigencia muscular y cardíaca. Por otro lado, los resultados demuestran la necesidad de realizar un descanso completo durante varios días para normalizar los parámetros fisiológicos alterados tras realizar una ultratrail. Aunque los corredores consideren que dejar de entrenar una semana es perder el tiempo, deben comprender que existe un daño agudo importante y que es imprescindible realizar un periodo de recuperación para poder seguir entrenando con garantías de éxito.

\section{Referencias bibliográficas}

Baird, Marianne F., Scott M. Graham, Julien S. Baker y Gordon F. Bickerstaff. 2012. "Creatine-kinase- and exercise-related muscle damage implications for muscle performance and recovery». Journal of Nutrition and Metabolism, 2012: 960363. https://doi.org/10.1155/2012/960363

Christensen, Dirk L., Diana Espino, Rocío Infante-Ramírez, Mónica S. CervantesBorunda, Rosa P. Hernández-Torres, Antonio E. Rivera-Cisneros y Jesper 
Kjaergaard. 2017. "Transient cardiac dysfunction but elevated cardiac and kidney biomarkers $24 \mathrm{~h}$ following an ultra-distance running event in Mexican Tarahumara». Extreme Physiology \& Medicine, 6(1): 3. https://doi.org/10.1186/s13728-017-0057-5

Dill, David Bruce y David L. Costill. 1974. "Calculation of percentage changes in volumes of blood, plasma, and red cells in dehydration». Journal of Applied Physiology, 37(2): 247-248. https://doi.org/10.1152/jappl.1974.37.2.247

Hoffman, Martin D. 2016. "State of the Science-Ultraendurance Sports». International Journal of Sports Physiology and Performance, 11(6): 831-832. https://doi.org/10.1123/ijspp.2016-0472

Holtzhausen, Lucy-Mae, Timothy Noakes, Bettina Kroning, Marné Klerk, Mimi Roberts y Robin Emsley. 1994. "Clinical and biochemical characteristics of collapsed ultra-marathon runners». Medicine and Science in Sports and Exercise, 26(9): 1095-1101.

Joslin, Jeremy, Robert Worthing, Trevor Black, William Grant, Timur Kotlyar y Susan Wojcik. 2014. "Analysis of weight change and Borg rating of perceived exertion as measurements of runner health and safety during a 6-day, multistage, remote ultramarathon». Clinical Journal of Sport Medicine: Official Journal of the Canadian Academy of Sport Medicine, 24(3): 245-250. https://doi.org/10.1097/JSM.0000000000000019

Kargotich, Stephen, Carmél Goodma, David Keast, Rod W. Fry, Peter Garcia-Webb, Patrick M. Crawford y Alan R. Morton. 1997. «Influence of exercise-induced plasma volume changes on the interpretation of biochemical data following high-intensity exercise». Clinical Journal of Sport Medicine: Official Journal of the Canadian Academy of Sport Medicine, 7(3): 185-191.

Khodaee, Morteza, John Spittler, Karin VanBaak, Bradley Changstrom y John Hill. 2015. «Effects of Running an Ultramarathon on Cardiac, Hematologic, and Metabolic Biomarkers». International Journal of Sports Medicine, 94(11): 867871. https://doi.org/10.1055/s-0035-1550045

Kim, Hyo Jeong, Yoon Hee Lee y Chang Keun Kim. 2007. «Biomarkers of muscle and cartilage damage and inflammation during a $200 \mathrm{~km}$ run». European Journal of Applied Physiology, 99(4), 443-447. https://doi.org/10.1007/s00421-006-0362-y

Kim, Young-Joo, Chul-Hyun Kim, K-A Shin, A-C Kim, Y-H Lee, C-W Goh y Yongbum Park. 2012. "Cardiac markers of EIH athletes in ultramarathon». International Journal of Sports Medicine, 33(3): 171-176. https://doi.org/10.1055/s-00311285930

Knechtle, Beat y Pantelis Nikolaidis. 2018. «Physiology and Pathophysiology in UltraMarathon Running». Frontiers in Physiology, 9: 264. https://doi.org/10.3389/fphys.2018.00634

Son, Hee Jeong, Yoon Hee Lee, Jeong Hoon Chae y Chang Keun Kim. 2015. «Creatine kinase isoenzyme activity during and after an ultra-distance $(200 \mathrm{~km})$ run». Biology of Sport, 32(4): https://doi.org/10.5604/20831862.1163384 